\title{
MAXIMISING QUALITY IN HEALTH SURVEYS: LESSONS FROM THE FIELD
}

\section{Matthew Gorringe and Lindy Latham NSW Health Survey Program}

The NSW Health Survey Program (HSP) has a goal to maximise the quality of the data it collects. This requires a comprehensive approach to quality control, which includes the implementation of procedures to ensure that minimum standards are met, the ongoing training of interviewers, and the motivation of interviewers to achieve high professional standards. Implicit in this approach is the notion that quality and productivity are not competing priorities. This article describes some of the mechanisms for quality control implemented by the HSP.

\section{INTERVIEWER TRAINING AND CODE OF CONDUCT}

When they join the HSP, all interviewers attend two fourhour training sessions that cover the theory and practice of telephone interviewing, the aims and content of the health surveys, and the NSW Health Survey Code of Conduct and Confidentiality Agreement. A written manual accompanies the training sessions.

The NSW Health Survey Code of Conduct and Confidentiality Agreement was developed specifically for the HSP to ensure that it complies with the codes and regulations regarding the protection of privacy and personal health information. The Code is an amalgamation of several professional codes, including the NSW Health Information Privacy Code of Practice, the Code of Professional Behaviour for the Market Research Society of Australia, and the Code of Ethics for Interpreters and Translators. ${ }^{1}$

The Code of Conduct establishes a guideline for an acceptable standard of performance and behaviour. The Code of Conduct also addresses data security and confidentiality issues relating to the conduct of health survey interviews, and issues pertaining to the storage of and access to survey data. All interviewers are required to sign the Confidentiality Agreement prior to starting to conduct interviews.

\section{VALIDATION OF INTERVIEWS}

HSP supervisors recontact a random sample of 10 per cent of households that have been interviewed, to verify that the interview has occurred, and that the data are being recorded correctly. This ensures a minimum standard of quality is met, but does not guarantee that all the questions are being administered in a consistent and appropriate manner. For example, it is not possible to gauge reliably whether interviewers are prompting the respondent with possible answers to questions, or if they are probing for greater detail in a manner that influences the response given.

\section{OBSERVATION OF INTERVIEWS}

For reasons of privacy, it is not possible or appropriate to monitor interviews by listening to conversations between interviewers and respondents. However, HSP supervisors regularly observe interviewers by sitting behind them to check that they are following the questionnaire instructions correctly. This is particularly useful for providing feedback on their performance to interviewers who are new to the HSP. However, the value of this method is limited because interviewers know when they are being observed, and may change their habits accordingly.

\section{COMMITMENTTO QUALITY}

Because of the limitations of the procedural methods of quality control, achievement of more than a minimum level of quality in health survey fieldwork relies on the integrity and commitment of the interviewers themselves. The commitment to quality demonstrated both by the supervisors and senior staff involved in the HSP is important in fostering a 'desire to achieve' in the interviewers.

The management style of the HSP is based on the concept of Total Quality Management. Elements of this approach include; adopting meaningful measures of quality, adopting modern training methods, statistically validating quality, and teamwork. ${ }^{2}$ An integral part of this approach is the concept of intrinsic motivation versus extrinsic motivation (see Box). According to this theory, people have to want to do their work more efficiently and effectively through self-motivation and pride in their work. If the motivation is only extrinsic, the drive for improvement or quality will disappear as soon as the external reinforcement mechanisms cease to exist or be effective. ${ }^{3}$

\section{Extrinsic motivation \\ The motivation to perform an activity as an external consequence of the activity (such as social approval, status, material rewards). \\ Intrinsic motivation \\ The motivation to perform an activity for its own sake (such as pleasure, mastery, curiosity, or fun).}

Maintaining the intrinsic motivation of the interviewer relies on their high regard for the organisation. The recruitment process undertaken by the HSP, ${ }^{1}$ although standard for the public sector, differs significantly from the usual process used for employing market researchers. The rigour of the process, and the time spent in recruiting and training, demonstrates to the applicants the value of the organisation that they are seeking to join, and assists in instilling purpose and motivation. 
To further build and maintain this motivation, management staff of the HSP give interviewers regular feedback on their performance including response rates, queries received about the survey, and reports on previous data collected, and the usefulness of that data.

A survey of interviewers employed by the HSP recently carried out within the HSP highlighted that it is not only the task that motivates interviewers to perform, but also the environment of which they are a part. The factors mentioned by interviewers as contributing to interview quality include:

- '... a happy relaxed environment';

- '... all supervisors are helpful in attending promptly to any queries ... team spirit is great';

- 'knowing the importance of our part or contribution to the quality of the data'.

\section{CONCLUSIONS}

The NSW Health Survey Program has adopted a holistic approach to quality control, involving both procedural quality control measures and methods to build interviewer motivation and performance. Further examination of specific aspects of the HSP's operation, such as reasons for staff turnover, will enable HSP staff to further refine its human resource management.

\section{REFERENCES}

1. Williamson M, Baker D, Jorm L. The NSW Health Survey Program: Overview and methods, 1996-2000. Sydney: NSW Department of Health, 2001.

2. Nankervis A, Compton R, McCarthy T. Strategic Human Resource Management, 2nd edition. South Melbourne: Thomas Nelson Australia, 1996.

3. Dubnick C, Williams, J. The people side of TQM. Healthcare Forum, Sept-Oct 1992; 35(5): 54-61. 蛋

\section{CONTINUOUS DATA COLLECTION UNDERTHE NSW HEALTH SURVEY PROGRAM-WHAT WILL IT MEAN?}

\section{Margo Eyeson-Annan \\ NSW Health Survey Program \\ NSW Department of Health}

From January 2002, continuous data collection under the NSW Health Survey Program (HSP) will commence. The new continuous Health Survey data collection will focus on providing information to support the public health priority areas outlined in Healthy People 2005-New directions for public health in NSW: ${ }^{1}$ social determinants of health, individual or behavioural determinants of health, major health problems, population groups with special needs, settings, partnerships, and infrastructure. This article describes the proposed changes to the HSP and how they will affect users and collaborators.

\section{CHANGESTOTHE HEALTH SURVEY PROGRAM}

Six major changes from the existing HSP are proposed:

- there will be a five-year development, collection and reporting plan;

- the sample will include the whole population (from birth upwards), with parents or carers of children under 16 being interviewed;

- interviews will be conducted throughout the whole year, instead of for the discrete period for each survey;

- at least 2,000 interviews will be completed each month (except for a month over Christmas and New Year period), giving a total of 22,000 interviews each year divided equally among the NSW area health services;

- interviews will be conducted in all area health services all year round;
- there will be improved reporting, using automated and interactive reporting facilities.

\section{Survey development}

The continuous Health Survey data collection will continue to use the methodologies developed since 1996 by the Epidemiology and Surveillance Branch of the NSW Department of Health. ${ }^{2}$

The existing questionnaires from the 1997 and 1998 NSW Health Surveys, ${ }^{3} 1999$ Older People's Health Survey, ${ }^{4}$ and 2001 Child Health Survey, will be combined and rationalised to develop core sets of questions. These will include questions on demographics and key indicators for all ages, including the access to and satisfaction with health services; plus core questions specific to each age group (infants, toddlers, primary school children, youth, adults, and older persons). Table 1 presents the topic areas that will be covered.

As well as these core questions, the questionnaire will include modules of questions that explore particular issues, which will be delivered to only a proportion of the population and/or for discrete periods of time. Where possible, these questions will be drawn from those used in previous surveys of the HSP for the relevant topic. The new questionnaire will also include area health servicespecific questions and questions to address emergent issues.

As far as possible, the continuous survey tool will be kept to a maximum of 20 minutes interview time, with time allocated for each type of question as follows: 